\title{
Hemorragia subaracnoidea como complicación de trombólisis endovenosa en una paciente con accidente cerebro vascular isquémico.
}

Subarachnoid hemorrhage as a complication associated with thrombolysis in a patient with ischemic stroke.

\begin{abstract}
Mayra Rojas-Benites ${ }^{1,2, a}$, Leily Campos-Flores ${ }^{2, b}$, Juan Cancino-Díaz ${ }^{2, c}$, Eduardo Carranza-Jordán ${ }^{2, d}$,
\end{abstract} Leonardo Castillo-Zegarra ${ }^{2, e}$, José Cruz-Rodríguez 2,f, Waldir De la Cruz-Araujo ${ }^{2, g}$.

Identificación ORCID:

Mayra Rojas-Benites: ORCID ID: 0000-0002-7783-1000

Leily Campos-Flores: ORCID ID: 0000-0002-7419-2266

Juan Cancino-Díaz: ORCID ID: 0000-0001-7886-4923

Eduardo Carranza-Jordán: ORCID ID: 0000-0001-6320-4236

Leonardo Castillo-Zegarra: ORCID ID: 0000-0001-7599-0414

José Cruz-Rodríguez: ORCID ID: 0000-0003-4281-5956

Waldir De la Cruz-Araujo: ORCID ID: 0000-0002-4584-4472

\section{RESUMEN}

La hemorragia subaracnoidea es una complicación muy infrecuente del tratamiento trombolítico. Se presenta el caso de una paciente mestiza de 76 años portadora de marcapasos que ingresó a emergencia con manifestaciones de trastorno de conciencia, dificultad para la marcha y disartria. Como antecedentes relevantes, reportó hipertensión arterial mal controlada, hipotiroidismo y numerosas intervenciones quirúrgicas. Sobre la base de Tomografia cerebral Espiral Multicorte (TEM) sin contraste y de las manifestaciones clínicas, se estableció un diagnóstico de accidente cerebrovascular (ACV) isquémico. Se inició inmediatamente terapia trombolítica con alteplasa (rt-PA) y a las 3 horas postratamiento se realizó una TEM cerebral control sin contraste que permitió identificar hemorragia subaracnoidea (HSA) como complicación del tratamiento. Un Angio-TEM efectuado 24 horas después confirmó $\mathrm{ACV}$ isquémico con transformación hemorrágica, sin presencia de aneurisma roto. La paciente fue admitida a la Unidad de Cuidados Intensivos (UCI), quedando en observación y con control permanente de signos vitales; a los 20 días fue dada de alta. La identificación y reporte de esta muy poco usual complicación contribuyen a una mejor comprensión y diagnóstico del caso.

PALABRAS CLAVE: Accidente cerebrovascular, isquemia encefálica, terapia trombolítica, hemorragia subaracnoidea.

\footnotetext{
Hospital Regional Docente de Trujillo. Trujillo, Perú.

Facultad de Medicina, Universidad Nacional de Trujillo. Trujillo, Perú.

Médico Neurólogo.

Estudiante de Medicina.

Estudiante de Medicina.

Estudiante de Medicina.

Estudiante de Medicina.

f Estudiante de Medicina.

g Estudiante de Medicina.
} 


\section{SUMMARY}

Subarachnoid hemorrhage is a very rare complication of thrombolytic therapy. We report the case of a 76-yearold mestizo woman with a pacemaker, admitted to the emergency room due to compromised consciousness, walking difficulties and dysarthria. She reported a history of poorly controlled hypertension, hypothyroidism, and numerous surgical interventions. Based on a non-contrast Multislice Computed Tomography (MSCT) of the brain and the clinical manifestations, a diagnosis of ischemic stroke was established. Thrombolytic therapy with alteplase was immediately started and another non-contrast MSCT brain scan, performed three hours later, identified a subarachnoid hemorrhage as a treatment complication. Twenty-four hours later, a CT angiography confirmed an ischaemic stroke with hemorrhagic transformation, without the presence of a ruptured aneurysm. The patient was admitted to ICU, remaining in observation with permanent control of vital signs, and discharged after 20 days. the patient was discharged. The identification and reporting of this unusual complication contribute to a better understanding and diagnosis of the case.

KEYWORDS: Stroke, brain ischemia, thrombolytic therapy, subarachnoid hemorrhage.

\section{INTRODUCCIÓN}

La trombólisis endovenosa con alteplasa es el pilar en el tratamiento del ACV isquémico dentro de las 4,5 horas posteriores al inicio de los síntomas neurológicos definidos (1). Dicho manejo está relacionado a la condición del paciente y al grado de severidad de la isquemia, los cuales se reconocen mediante la evaluación neurológica y el estudio de neuroimagen. La evaluación cuantitativa del déficit neurológico del paciente con ACV se realiza a través de la escala NIHSS (National Institute of Health Stroke Scale) $(1,2)$. En neuroimagen, la TEM sin contraste se considera suficiente para el manejo inicial y en pacientes fuera del tiempo de ventana de 4,5 horas se emplean las imágenes de perfusión basadas en TEM o RM para seleccionar el tratamiento $(1,3)$.

Sin embargo, su aplicación debe tener en cuenta las posibles complicaciones que puede traer, tales como hemorragia intracerebral sintomática (5-7\%), angioedema (1-8\%) y hemorragia sistémica $(1,2)$. En este contexto, reportamos el caso de una paciente adulto mayor que desarrolló hemorragia subaracnoidea como complicación al tratamiento trombolítico de un $\mathrm{ACV}$ isquémico.

\section{PRESENTACIÓN DEL CASO}

Mujer de 76 años procedente de Trujillo, usuaria de marcapasos desde hace 5 años por bradiarritmia, con antecedentes de hipotiroidismo controlado, HTA no controlada y múltiples intervenciones quirúrgicas. No refiere alergia a medicamentos. Ingresa a emergencia por presentar desde hace 30 minutos trastorno de conciencia, dificultad para la marcha y disartria. Al ingreso se registró PA: 173/118 mmHg, FC: 128 lpm,
FR: 28 rpm, ECG: 11 puntos. Peso: 90 kg Talla: 160 $\mathrm{cm}$. Presenta somnolencia y hemiplejia izquierda con parálisis facial central izquierda. Se solicitó TEM cerebral sin contraste, el cual reveló foco isquémico a nivel parietal derecho (figura 1). Se estableció como diagnóstico $\mathrm{ECV}$ isquémico. A las 2 horas, presentó PA: 183/72 mmHg, FC: 128 lpm, FR: 28 rpm, ECG: 13 puntos. Se optó por tratamiento trombolítico con Alteplasa $70 \mathrm{mg}$ E.V. (10 mg en 5 minutos seguido de $60 \mathrm{mg}$ en 60 minutos).

A las $3 \mathrm{~h}$, la escala NIHSS fue de 19 puntos, asociada a hemiplejía izquierda, lateralización de la mirada a la derecha y compromiso severo de conciencia. Se indicó

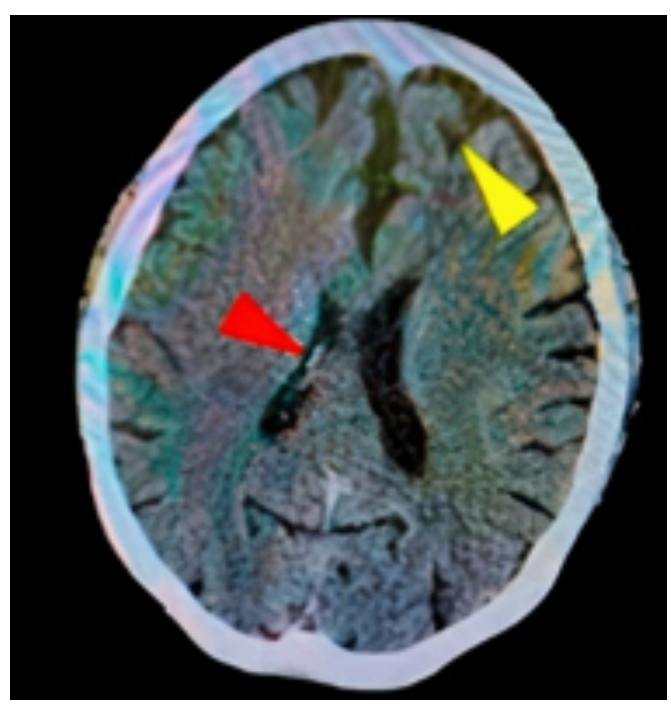

Se evidencia infarto en territorio de la ACM derecha. Calcificaciones a nivel de ambos ganglios basales con arterias vertebrales calcificadas (cabeza flecha roja), cambios tróficos a nivel del parénquima cerebral (cabeza flecha amarillo).

Figura 1. TEM de cráneo sin contraste (13 horas antes de uci) corte axial. 
una TEM cerebral sin contraste control, encontrándose foco isquémico en región parietal derecha, asociado a hemorragia subaracnoidea (figura 2). Luego de permanecer 7 horas en emergencia, ingresa a UCI. A las 12 horas se registra PA de $181 / 85 \mathrm{mmHg}$, asociado a taquicardia, taquipnea y febrícula $\left(37,6^{\circ} \mathrm{C}\right)$. A las $24 \mathrm{~h}$ se realiza una TEM cerebral y una angio-TEM que reveló un ECV isquémico con transformación

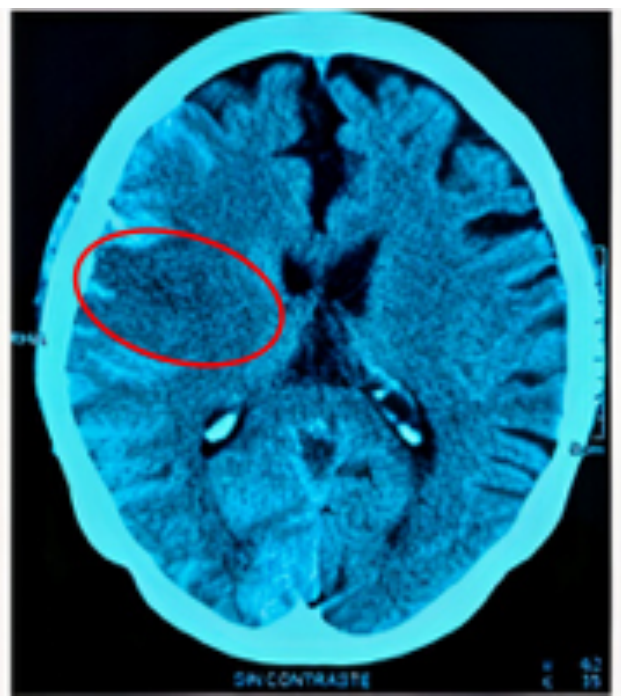

Foco isquémico (círculo rojo) en parietal derecho de bordes definidos asociado a hemorragia subaracnoidea.

Figura 2. TEM cerebral sin contraste control (12 horas antes de UCI) de corte axial.

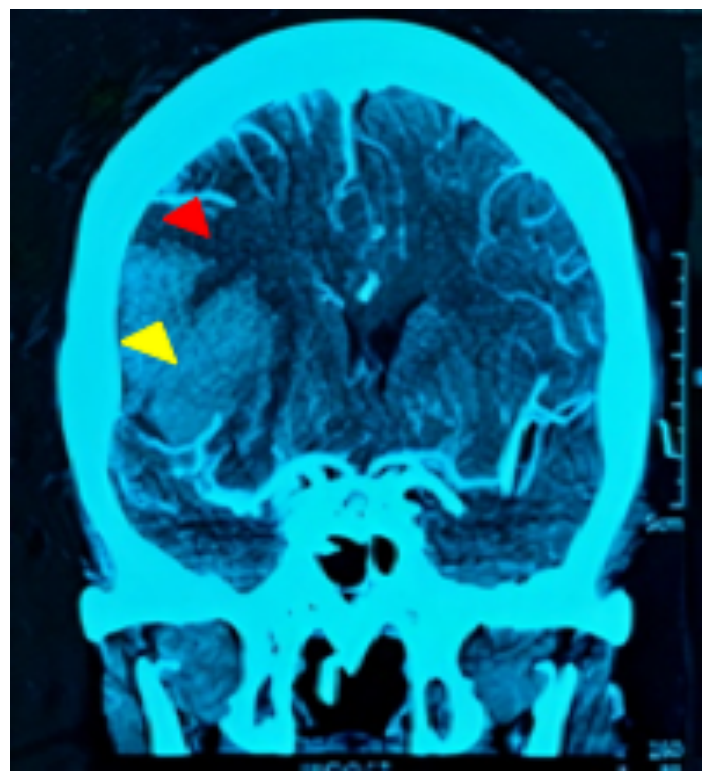

Área hipodensa (cabeza flecha roja) de bordes definidos asociado a disminución de la vascularización, con hematoma intraparenquimal derecho (cabeza flecha amarilla).

Figura 3. ANGIOTEM cerebral (día 2 de UCI). Corte coronal.

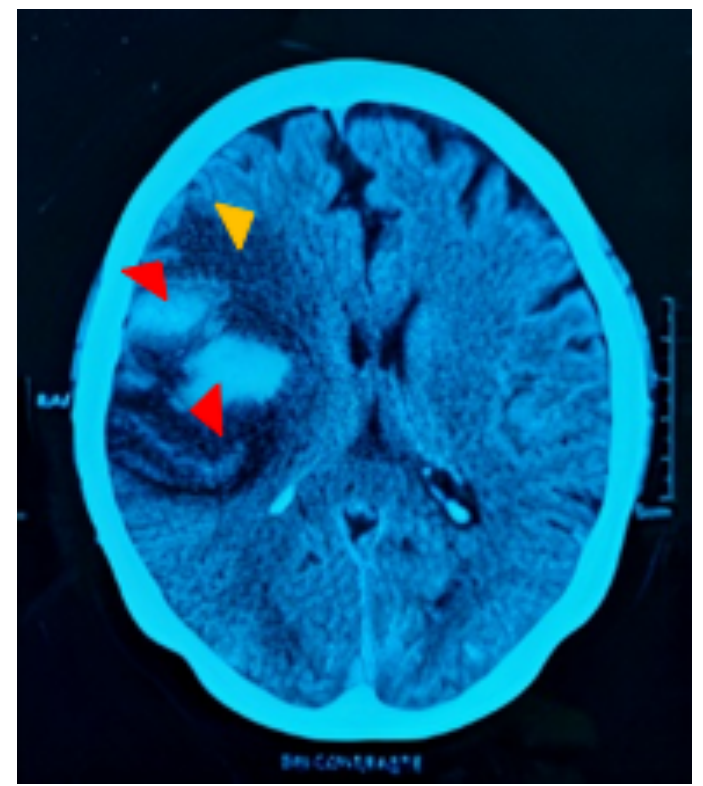

Amplia área isquémica (cabeza flecha roja) y zonas hiperdensas que corresponden al sangrado intraparenquimal a nivel parietal derecho (cabeza flecha naranja).

Figura 4. TEM cerebral sin contraste (día 13 de UCI). Corte axial.

hemorrágica post trombólisis, sin presencia de aneurisma roto (figura 3). La paciente permaneció en UCI en ventilación mecánica. A los 10 días, se realizó una TEM control que reveló menor volumen de hematoma en comparación al estudio tomográfico previo (figura 4). A los 20 días tras su ingreso a UCI fue dada de alta

\section{DISCUSIÓN}

El tratamiento trombolítico con rt-PA por vía EV es recomendado en pacientes con infarto cerebral agudo de evolución menor a 4,5 horas según la Sociedad Española de Neurología y la AHA/ASA $(4,5)$. La administración oportuna de rt-PA se asocia a un mejor pronóstico en la calidad de vida del paciente y disminuye de manera considerable el riesgo de mortalidad. Sin embargo, involucra cierto riesgo de hemorragia intracerebral $(2,6,7)$.

La paciente en el presente caso inició el tratamiento con alteplasa intravenosa a las 2,5 horas de haber iniciado el cuadro, lo que está dentro del tiempo descrito como adecuado. Asimismo, cumple con el criterio de inclusión de diagnóstico clínico de infarto cerebral agudo que causa déficit neurológico apreciable (2), pues a su ingreso presentó trastorno de conciencia, dificultad para la marcha, disartria y hemiplejía izquierda. Las guías mencionan que 
el paciente es elegible para el tratamiento desde los 18 años, estando la paciente dentro del grupo etario aceptable (4). El ensayo del grupo de estudio rt-PA del Instituto Nacional de Trastornos Neurológicos y Accidentes Cerebrovasculares (NINDS) halló que el tratamiento durante una hora bajo condiciones estrictas aumentó la probabilidad de una discapacidad mínima o nula a los tres meses en al menos en un 30\% en los diferentes grupos etarios (8).

Con respecto a los criterios de exclusión, la paciente no presentó hemorragia intracraneal en la TEM tomada previamente a la administración del rt-PA. No presenta antecedentes de infarto cerebral en meses previos o de hemorragia intracraneal ni tratamiento con anticoagulantes orales. El tratamiento no fue indicado por un médico neurólogo con experiencia en manejo de ictus, al contrario de lo que recomienda la guía clínica española (4). Este hecho puede explicar la falta de estimación del puntaje en la escala NIHSS, que es un criterio de exclusión si se presenta un valor $>25$ (infarto severo) $(2,4,5,9)$. Al momento de la aplicación, la presión diastólica era mayor a $110 \mathrm{mmHg}(118 \mathrm{mmHg})$ previa administración de antihipertensivos, lo que constituye un criterio de exclusión (5). En pacientes con la presión arterial elevada y que sean candidatos para recibir tratamiento con alteplasa, se debe reducir la $\mathrm{PA}<185 / 110 \mathrm{mmHg}$, antes de iniciar el tratamiento con fibrinolítico IV. Se recomienda que PA sea $\leq 180 / 105 \mathrm{mmHg}$ durante las primeras 24 horas después del tratamiento (I B-NR). De no corregir estos valores, el riesgo de hemorragia es mayor tras la administración de alteplasa IV, por lo que estas mediciones deben ir en constante manejo antes, durante y después de la terapia fibrinolítica, cada 15 minutos durante las primeras 2 horas, luego cada 30 minutos durante 6 horas y cada hora, hasta completar las 24 horas post tratamiento (5).

Con respecto a la dosis estándar de alteplasa $(0,9$ $\mathrm{mg} / \mathrm{kg}$ ), no se conoce con seguridad si esta es la más eficaz (10). Se observó que, al administrarla en poblaciones como los adultos mayores, aquellos con enfermedades metabólicas y asiáticos tenían un mayor riesgo de hemorragias. Se investiga en la actualidad si el reducir la dosis es posible manteniendo la eficacia (10). La dosis de alteplasa administrada en el caso fue de $70 \mathrm{mg}$, menor a la dosis estándar, presentando una hemorragia subaracnoidea como complicación al tratamiento, sin embargo, estudios reportaron que una dosis reducida de alteplasa no era inferior en eficacia a la dosis estándar y se asociaba a un riesgo significativamente menor de hemorragia intracraneal $(10,11)$. Por otro lado, en un estudio de cohorte, según Aulicky P. et al, los pacientes tratados con dosis mayores y menores de rt-PA presentaron una tendencia a un peor desenlace y mayor incidencia de hemorragia parenquimal, aunque no fueron significativamente diferentes bajo el análisis estadístico (12).

Dentro de las complicaciones post-tratamiento trombolítico con alteplasa se ha considerado a la hemorragia intracerebral como una de las más importantes, pero a medida en que aumenta de riesgo absoluto en exceso difiere según el tiempo de tratamiento, la edad o la gravedad del accidente cerebrovascular. Se ha reportado que el alteplasa puede ocasionar una hemorragia intraparenquimal en un $6.8 \%$ (7). Otro punto a considerar sería que la hemorragia intraparenquimal pueda extenderse hacia el espacio subaracnoideo por la contigüidad de las estructuras anatómicas y dar la impresión de ser una hemorragia subaracnoidea por una lesión vascular, éste último fue descartado con el estudio de una Angio-TEM cerebral.

Sin embargo, la descripción o el reporte del desarrollo de una hemorragia subaracnoidea tras el tratamiento trombolítico con alteplasa post-ictus es muy infrecuente (13). Revisamos la literatura y encontramos 3 casos semejantes a lo reportado. Según Lagares A, et al., presentaron un caso de hemorragia subaracnoidea después de usar rt-PA como tratamiento en un cuadro de isquemia miocárdica (14). Chou C, et al. informaron un cuadro clínico similar al caso presentado, en el cual aplican rt-PA siguiendo el protocolo estándar, 130 minutos después del inicio de los síntomas. En esta investigación por primera vez se reportó esta complicación infrecuente y se propuso a la disección espontánea de la porción intracraneal de la arteria carótida interna con extensión hasta la arteria cerebral media como mecanismo fisiopatológico responsable de la aparición de la hemorragia subaracnoidea (13). Gopal M, et al. informaron que 5 pacientes con $\mathrm{ACV}$ isquémico agudo que recibieron activadores del plasminógeno tisular endovenoso (IV tPA), fueron diagnosticados con endocarditis infecciosa, de los cuales 2 pacientes desarrollaron complicaciones hemorrágicas, donde uno de ellos presentó hemorragia subaracnoidea debido a la ruptura del aneurisma cerebral micótico (15).

En el presente caso, no se detectó en la angio-TEM cerebral la presencia de aneurisma o lesión vascular, teniendo en cuenta que este examen es altamente sensible; por lo que sugerimos que para la localización de una lesión vascular probable como origen de 
Hemorragia subaracnoidea como complicación de trombólisis endovenosa en una paciente con accidente cerebro vascular.

la hemorragia subaracnoidea se debió realizar una panangiografía cerebral, la cual tiene mayor sensibilidad para detectar la localización y tamaño de aneurismas cerebrales $(16,17,18)$. En este contexto, consideramos que el presente trabajo corresponde a uno de los escasos reportes de esta complicación del uso de alteplasa como parte del tratamiento del $\mathrm{ACV}$ isquémico, siendo este el motivo por el que la manifestación de hemorragia subaracnoidea debe ser considerada por el personal de salud al momento de administrar el fármaco (13).

\section{Apreciaciones finales}

Se reportó el cuadro clínico de una hemorragia subaracnoidea como complicación del tratamiento trombolítico en un ACV isquémico asociado a un mal control de la PA antes, durante y después del tratamiento, la cual, debió ser considerado por el personal de salud al momento de administrar el fármaco.

Se destaca esta complicación a pesar de administrarle una dosis baja de alteplasa. En lo que respecta al manejo que se realizó de la paciente, en los estudios de neuroimagen no se detectó el origen de la lesión vascular inicial, por lo que se debió realizar la panangiografía para identificar la causa final de la hemorragia subaracnoidea.

\section{Correspondencia:}

Mayra Janett Rojas Benites.

Hospital Regional Docente de Trujillo, Servicio de Neurología. Av. Mansiche 795. Cod. Postal: 13011. Trujillo.

Correo electrónico: mayrarojasneuro@gmail.com

Financiamiento: Autofinanciado.

Conflictos de interés: Los autores declaran no tener conflictos de interés.

\section{REFERENCIAS BIBLIOGRÁFICAS:}

1. Phipps $\mathrm{M}$, Cronin C. Management of acute ischemic stroke. BMJ. 2020;368: 16983. DOI:10.1136/bmj. 16983

2. Oliveira J, Samuels O. Intravenous thrombolytic therapy for acute ischemic stroke: Therapeutic use. UpToDate. 2020. (Citado el 7 de octubre de 2020). Disponible en: https://www.uptodate.com/contents/ intravenous-thrombolytic-therapy-for-acuteischemic-stroke-therapeutic-use. DOI: 10.1056/
NEJMct 1007370

3. Campbell B, Khatri P. Stroke. The Lancet. 2020; 396 (10244): $\quad 129-42 . \quad$ DOI: $10.1016 / \mathrm{s} 0140-$ 6736(20)31179-x

4. De Leciñana M, Egido J, Casado I, Ribó M, Dávalos A, Masjuan J, et al. Guía para el tratamiento del infarto cerebral agudo. Neurología. 2014; 29(2): 102-22. DOI: 10.1016/j.nrl.2011.09.012

5. Powers W, Rabinstein A, Ackerson T, Adeoye O, Bambakidis N, Becker K. Guidelines for the Early Management of Patients With Acute Ischemic Stroke: 2019 Update to the 2018 Guidelines for the Early Management of Acute Ischemic Stroke: A Guideline for Healthcare Professionals From the American Heart Association/American Stroke Association. Stroke. 2019; 50 (12): e344-e418. DOI: 10.1161/str.0000000000000211

6. Oliveira J, Mullen MT. Initial assessment and management of acute stroke. UpToDate. 2020. (Citado el 7 de julio de 2021). Disponible en: https://www.uptodate.com/contents/initialassessment-and-management-of-acutestroke?topicRef $=16134 \&$ source $=$ see

7. Whiteley W, Emberson J, Lees K, Blackwell L, Albers G, et al. Risk of intracerebral haemorrhage with alteplase after acute ischaemic stroke: a secondary analysis of an individual patient data meta-analysis. Lancet Neurol. 2016; 15: 925-33. DOI: $10.1016 / \mathrm{S} 1474-4422(16) 30076-\mathrm{X}$

8. The NINDS T-PA Stroke Study Group. Generalized efficacy of t-PA for acute stroke. Subgroup analysis of the NINDS t-PA Stroke Trial. Stroke. 1997; 28: 211925. DOI: 10.1161/01.str.28.11.2119

9. Choi J, Kim J, Han M, Lee S, Kang K, Park J, et al. Utility of Items of Baseline National Institutes of Health Stroke Scale as Predictors of Functional Outcomes at Three Months after Mild Ischemic Stroke. J Stroke Cerebrovasc Dis. 2017; 26 (6): 130613.DOI: 10.1016/j.jstrokecerebrovasdis.2017.01.027.

10. Moussaddy A, Demchuk A, Hill M. Thrombolytic therapies for ischemic stroke: Triumphs and future challenges. Neuropharmacology. 2018; 134: 272-79. DOI:10.1016/j.neuropharm.2017.11.010

11. Cheng J, Zhang X, Cheng L, Li G, Zhang L, Ji K, et al. Tissue Plasminogen Activator in Acute Ischemic Stroke: A Systematic Review and MetaAnalysis. Journal of Stroke and Cerebrovascular Diseases. 2018; 27(2): 381-90. DOI: 10.1016/j. jstrokecerebrovasdis.2017.09.014

12. Aulicky P, Rabinstein A, Seet R, Neumann J, Mikulik R. Dosing of Tissue Plasminogen Activator Often Differs from $0.9 \mathrm{mg} / \mathrm{kg}$, but Does Not Affect the Outcome. Journal of Stroke and Cerebrovascular Diseases. 2013; 22(8): 1293-97. DOI: 10.1016/j. jstrokecerebrovasdis.2012.10.010

13. Chou C, Lin Y, Po H, Chang C. Subarachnoid 
hemorrhage: a rare complication after intravenous thrombolysis in an ischemic stroke patient. Am J Emerg Med. 2010; 28 (8): 984. e1-3. DOI: 10.1016/j. ajem.2009.12.024

14. Lagares A, Gomez P, Lobato R, Alén J, Campollo J, Benito-León J. Cerebral aneurysm rupture after r-TPA thrombolysis for acute myocardial infarction. Surg Neurol. 1999; 52 (6): 623-6. DOI: 10.1016/ s0090-3019(99)00147-0

15. Gopal M, Lakhani S, Lee VH. Intravenous Thrombolysis in Acute Ischemic Stroke Patients with Unsuspected Infective Endocarditis. J Stroke Cerebrovasc Dis. 2021; 30(3):105502. DOI: 10.1016/j.jstrokecerebrovasdis.2020.105502
16. Steiner T, Juvela S, Unterberg A, Jung C, Forsting M, Rinkel G. European Stroke Organization Guidelines for the Management of Intracranial Aneurysms and Subarachnoid Haemorrhage. Cerebrovasc Dis. Cerebrovasc Dis. 2013; 35 (2): 93 112. DOI: $10.1159 / 000346087$

17. Barinagarrementería F, Dávila L, López M, Orozco A. Neurología elemental. New York: Elsevier Health Sciences; 2017.

18. Villatoro A. Manual de medicina de urgencias. Ciudad de Mexico: Editorial El Manual Moderno; 2013.

Recibido: 05/08/2021

Aceptado: 27/11/2021 